\title{
RESONANCE AND BOUND STATES IN PHOTONIC CRYSTAL SLABS*
}

\author{
STEPHEN P. SHIPMAN ${ }^{\dagger}$ AND STEPHANOS VENAKIDES ${ }^{\ddagger}$
}

\begin{abstract}
Using boundary-integral projections for time-harmonic electromagnetic (EM) fields, and their numerical implementation, we analyze EM resonance in slabs of two-phase dielectric photonic crystal materials. We characterize resonant frequencies by a complex Floquet-Bloch dispersion relation $\omega=W(\beta)$ defined by the existence of a nontrivial nullspace of a pair of boundary-integral projections parameterized by the wave number $\beta$ and the time-frequency $\omega$. At resonant frequencies, the crystal slab supports a source-free EM field. We link complex resonant frequencies, where the imaginary part is small, to resonant scattering behavior of incident source fields at nearby real frequencies and anomalous transmission of energy through the slab. At a real resonant frequency, the source-free field supported by the slab is a bound state. We present numerical examples which demonstrate the effects of structural defects on the resonant properties of a crystal slab and surface waves supported by a dielectric defect.
\end{abstract}

Key words. photonic crystal, boundary integral, Calderón's projection, resonance, dispersion relation, bound state, scattering, surface wave

AMS subject classifications. $78,45,65$

DOI. $10.1137 /$ S0036139902411120

1. Overview. Photonic crystals are material structures with spatially periodic electromagnetic (EM) properties. A two-dimensional (2D) dielectric photonic crystal slab (Figure 1.1) has dielectric permittivity that does not vary in the $z$ direction, is constant beyond some finite value of $|x|$, and is periodic in $y$. The photonic crystal slabs in our study consist of an array of circular homogeneous rods embedded in a matrix of a contrasting dielectric permittivity.

It is well known that photonic crystals may act as resonators. In previous work [1], [2], we investigated resonant behavior in photonic crystal slabs. In particular, a periodic channel defect in a slab whose period cell is shown in Figure 7.3(3b) below resulted in the appearance of narrow ranges of frequency values over which the steadystate field in the crystal exhibited amplitudes that were many times greater than the amplitude of the polarized, time and space harmonic incident EM source field [2]. The dielectric materials that we consider have no losses or gains; thus the phenomenon is due solely to resonant behavior in the scattering by the crystal. Over these narrow frequency ranges, the transmission of energy through the slab is either enhanced or inhibited, producing "spikes" in the transmission coefficient (as in Figures 7.1(5) and 7.3(3a) below). The exploitation of photonic crystal resonances in the engineering of photonic devices has received much attention in the literature in recent years. See, for example, [3] and [4] for applications to filters and transmission enhancement. The connection between the structure of transmission dips and properties of quasi-guided eigenmodes is investigated in [5] for square-patterned slabs on a substrate.

${ }^{*}$ Received by the editors July 10, 2002; accepted for publication (in revised form) March 25, 2003; published electronically November 19, 2003.

http://www.siam.org/journals/siap/64-1/41112.html

${ }^{\dagger}$ Department of Mathematics, Louisiana State University, Baton Rouge, LA 70803 (shipman@ math.lsu.edu).

${ }^{\ddagger}$ Department of Mathematics, Box 90320, Duke University, Durham, NC 27708-0320 (ven@math. duke.edu). The research of this author was supported by grants ARO-DAAD19-99-1-0132 and NSF DMS-0207262. 


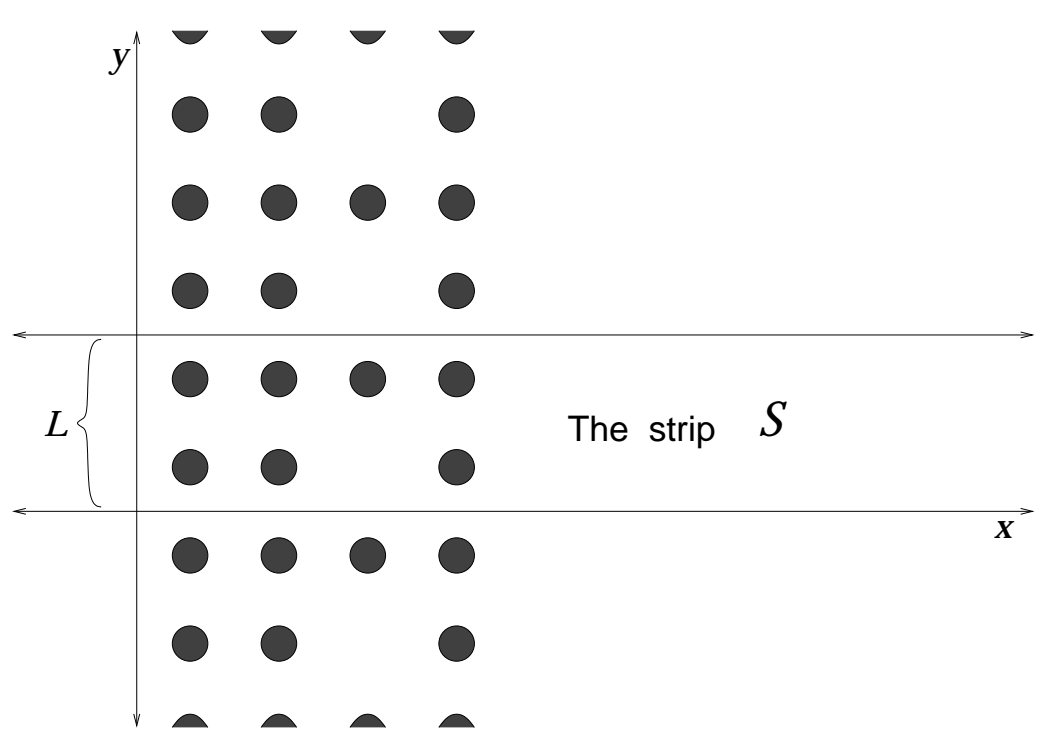

FIG. 1.1. A cross section of a photonic crystal slab consisting of an array of homogeneous dielectric rods standing perpendicular to the $x y$-plane. The rod structure is periodic in the $y$ direction, with period $L$, and extends indefinitely as $y \rightarrow \pm \infty$. The rod structure is finite in the $x$-direction. Exterior to the rods is a homogeneous material of contrasting dielectric permittivity extending to infinity to the right and left. Pseudoperiodic fields in the plane can be analyzed in the strip $\mathcal{S}=\{(x, y):-\infty<x<\infty, 0 \leq y \leq L\}$ consisting of a single period of the dielectric permittivity function.

A connection between resonant frequencies and proper eigenvalues is known for Helmholtz resonators. Beale [6] shows that the (complex) resonant frequencies of a cavity in $\mathbb{R}^{3}$ with an opening converge to the eigenvalues (bound state frequencies) of the closed cavity as the opening disappears.

In the present study, we link resonant scattering behavior in dielectric photonic crystal slabs to certain complex frequencies with a small imaginary part at which the structure supports a source-free field (the term "source" is defined precisely in section 4). These are Bloch fields $\psi(x, y)=\tilde{\psi}(x, y) e^{i(\beta y-\omega t)}$, with $\tilde{\psi}(x, y)$ periodic in $y$ and real wave number $\beta$. If a source-free field exists for a real value of $\omega$, the structure sustains a traveling or standing wave along the slab that decays exponentially as $|x| \rightarrow$ $\infty$ so that the slab acts as a waveguide. We call such a field a localized field or a bound state, localization being in the strip $\mathcal{S}=\{(x, y):-\infty<x<\infty, 0 \leq y \leq L\}$, consisting of one period of the dielectric permittivity function (Figure 1.1). Frequencies at which the crystal slab supports a source-free field are called resonant frequencies, and they are described by a dispersion relation $\omega=W(\beta)$. We take $\Re(\omega)>0$ and prove that $\Im(W(\beta)) \leq 0$ with equality if and only if the corresponding source-free field is a bound state. If $\Im(W(\beta))<0$, then the field grows exponentially as $|x| \rightarrow \infty$, but decays in time.

In numerical experiments with several structures, we find isolated values of the wave number $\beta$ for which the frequency $\omega=W(\beta)$ appears to be real, giving rise to an isolated bound state. At nearby values of $\beta, W(\beta)$ attains an imaginary part, and sources at real frequencies near $\Re(W(\beta))$ produce resonant scattering behavior. At these frequencies, the transmission coefficient exhibits anomalous behavior (see Figure 7.1). We also find a wave number range over which we show that the imaginary part 
of the frequency is exactly zero (see Figure 7.2). Indeed, a slab nine rods thick yields a real branch of the dispersion relation; all but the first rod have equal radii, and the radius of the first rod is larger. The wave modes sustained by this structure are mainly supported on the larger rod, demonstrating that waves can exist on a defective surface of an otherwise perfect crystal.

Following the Floquet-Bloch theory, our mathematical investigation restricts the analysis to a single period of the dielectric permittivity considered as a function of $x$ and $y$. Thus the problem is posed on the strip $\mathcal{S}=\{(x, y):-\infty<x<\infty, 0 \leq y \leq L\}$, where $L$ is the period in the $y$ direction, as illustrated in Figure 1.1. Pseudoperiodic boundary conditions then apply to the fields: $\psi(x, L)=e^{i \beta L} \psi(x, 0)$ and $\partial_{y} \psi(x, L)=$ $e^{i \beta L} \partial_{y} \psi(x, 0)$ for all values of $x$.

We use boundary-integral projections of Calderón's type with pseudoperiodic Green's functions and Green's identities on the strip $\mathcal{S}$. In sections 3 and 4 we show how these projections give rise to a system of two coupled integral equations that relate the trace of the steady-state field and its normal derivative on the boundaries of the rods to the trace of the source field and its normal derivative. The latter fields constitute the forcing. The system is Fredholm of the second kind in the former fields; that is, the integral operator involved is a compact perturbation of the identity.

The existence of a resonant frequency requires the existence of a nullspace of the boundary-integral operator; the latter depends parametrically on the dielectric structure and the parameters $\beta$ (which we always assume to be real) and $\omega$. To locate resonant frequencies in section 7 , we discretize the integral operator and search for $(\beta, \omega)$ pairs for which it has an eigenvalue equal to zero. In this way, we calculate numerically the dispersion relations $\omega=W(\beta)$. In some cases, $\omega$ is a real function of $\beta$, so that the relation describes how the frequency of an $x$-localized wave traveling along the slab depends on its Bloch wave number. In other cases, $\omega$ is a complex function of $\beta$. We prove that, in this case, the corresponding fields become unbounded as $|x| \rightarrow \infty$ (they decay as $t \rightarrow \infty$ ), and therefore do not represent bound states. They do, however, force nearby real frequencies to exhibit resonant behavior. This phenomenon is examined also in [5] using a scattering matrix for square-patterned slabs.

2. Free pseudoperiodic Green's functions. We consider a lossless photonic crystal that consists of an array of dielectric rods, each with the same constant dielectric coefficient $\epsilon_{1}>0$, embedded in a matrix of a material with some other constant dielectric coefficient $\epsilon_{0}>0$. The rods stand perpendicular to the $x y$-plane and do not vary with $z$. The array is truncated to a finite width in the $x$-direction and extends periodically in the $y$-direction, with period $2 \pi$. Its planar cross section consists of a finite union $D$ of planar domains $D_{j}$ (the cross sections of the rods) with $C^{2}$ boundaries in the strip $\mathcal{S}=\{(x, y): 0<y<2 \pi\}$ that repeats periodically in the $y$-direction. We let $\partial \mathcal{S}$ have an inward-pointing normal vector, and we let $n(\mathbf{r})$ denote the outward-directed normal vector to the boundary $\partial D$ of $D$.

Let $\psi(x, y) e^{-i \omega t}$ be the out-of-plane component (electric or magnetic) of a polarized time-harmonic electromagnetic field with nondimensionalized frequency ${ }^{1} \omega$ in the photonic crystal structure (it is constant in the $z$-direction). The Maxwell equations then reduce to the Helmholtz equation

$$
\nabla^{2} \psi+\epsilon_{1} \omega^{2} \psi=0 \quad(\text { in } D), \quad \nabla^{2} \psi+\epsilon_{0} \omega^{2} \psi=0 \quad(\text { in } \mathcal{S} \backslash \bar{D})
$$

\footnotetext{
${ }^{1}$ We use $\omega$ for the reduced time-frequency in this paper; it is equal to our $k$ in [1] and [2]. It relates to the physical frequency $\Omega$ (cycles per time) and period $L$ by $\omega=\Omega L / c$, where $c$ is the speed of light.
} 
and the matching conditions

$$
\begin{gathered}
\lim _{h \rightarrow 0}(\psi(\mathbf{r}-h n(\mathbf{r}))-\psi(\mathbf{r}+h n(\mathbf{r})))=0, \\
\lim _{h \rightarrow 0}\left(\frac{\partial \psi(\mathbf{r}-h n(\mathbf{r}))}{\partial n(\mathbf{r})}-\nu \frac{\partial \psi(\mathbf{r}+h n(\mathbf{r}))}{\partial n(\mathbf{r})}\right)=0,
\end{gathered}
$$

on the boundary $(\mathbf{r} \in \partial D)$. In these equations, $\nabla^{2}=\partial^{2} / \partial x^{2}+\partial^{2} / \partial y^{2}, \nu=1$ in the electric polarization case, and $\nu=\epsilon_{1} / \epsilon_{0}$ in the magnetic polarization case.

We will consider Helmholtz fields $\psi$ that are pseudoperiodic in $y$. This means that, for some real number $\beta, \psi=e^{i \beta y} \tilde{\psi}$, where $\tilde{\psi}$ is periodic in $y$ with the same period $(2 \pi)$ as the crystal.

First, we present the fundamental pseudoperiodic solutions of the Helmholtz equation.

TheOREM 2.1. Let $\epsilon$ and $\beta$ be real numbers and $\omega$ a complex number such that, for all integers $m, \epsilon \omega^{2}-(m+\beta)^{2} \neq 0$. For each integer $m$, let $\mu_{m}$ be defined by

$$
\mu_{m}^{2}-(m+\beta)^{2}+\epsilon \omega^{2}=0,
$$

with $\Re\left(\mu_{m}\right)<0$ for all but a finite number of values of $m$. Then the series

$$
G(\mathbf{r})=-\frac{1}{4 \pi} \sum_{m=-\infty}^{\infty} \frac{1}{\mu_{m}} \exp \left(\mu_{m}|x|+i(m+\beta) y\right)
$$

converges and is of class $C^{\infty}$ for all $\mathbf{r}=(x, y) \in \mathbb{R}^{2} \backslash\{(0,2 \pi n): n \in \mathbb{Z}\}$ and

$$
\nabla^{2} G+\epsilon \omega^{2} G=-\sum_{n=-\infty}^{\infty} \delta(x, y-2 \pi n) e^{2 \pi n i \beta}
$$

where $\delta$ is the Dirac delta-function in $\mathbb{R}^{2}$ with unit impulse at the origin.

Proof. We first consider the case in which $\Re\left(\mu_{m}\right) \leq 0$ for all $m \in \mathbb{Z}$. Then the series defining $G(\mathbf{r})$ converges to a tempered distribution on $\mathbb{R}^{2}$. This is seen as follows: Let $\phi(x, y)$ be a function of Schwartz class. Then, for $m$ large enough so that $\Re\left(\mu_{m}\right)<0$, we have

$$
\begin{aligned}
\mid \iint \frac{1}{\mu_{m}} \exp \left(\mu_{m}|x|+i\right. & (m+\beta) y) \phi(x, y) d x d y \mid \\
\leq & \frac{1}{\left|\mu_{m}\right|} \int e^{\Re\left(\mu_{m}\right)|x|} \int|\phi(x, y)| d y d x \leq \frac{-2 A}{\left|\mu_{m}\right| \Re\left(\mu_{m}\right)},
\end{aligned}
$$

where $A$ is such that

$$
\sup _{(x, y) \in \mathbb{R}^{2}}\left(1+y^{2}\right)|\phi(x, y)|<A\left(\int\left(1+z^{2}\right)^{-1} d z\right)^{-1} .
$$

Since $\Re\left(\mu_{m}\right) / m=\mathcal{O}(1)$ as $m \rightarrow \infty$, the series for $G(\mathbf{r})$ converges to a tempered distribution.

Let $\widehat{G}(\mathbf{s})$, where $\mathbf{s}=\left(s_{1}, s_{2}\right)$, be the Fourier transform of $G(\mathbf{r})$ :

$$
\widehat{G}(\mathbf{s})=-\frac{1}{4 \pi} \sum_{m=-\infty}^{\infty} \frac{-2}{s_{1}^{2}+\mu_{m}^{2}} \delta\left(s_{2}-(m+\beta)\right) ;
$$




$$
\begin{gathered}
{\left[\left(\nabla^{2}+\epsilon \omega^{2}\right) G\right]^{\uparrow}(\mathbf{s})=\frac{1}{2 \pi} \sum_{m=-\infty}^{\infty} \frac{-s_{1}^{2}-(m+\beta)^{2}+\epsilon \omega^{2}}{s_{1}^{2}+\mu_{m}^{2}} \delta\left(s_{2}-(m+\beta)\right)} \\
=-\frac{1}{2 \pi} \sum_{m=-\infty}^{\infty} \delta\left(s_{2}-(m+\beta)\right) \\
\Longrightarrow \quad\left(\nabla^{2}+\epsilon \omega^{2}\right) G=-\frac{1}{2 \pi} \delta(x) \sum_{m=-\infty}^{\infty} e^{i(m+\beta) y} \\
=-\frac{1}{2 \pi} \delta(x) e^{i \beta y} \sum_{m=-\infty}^{\infty} e^{i m y}=-\delta(x) e^{i \beta y} \sum_{n=-\infty}^{\infty} \delta(y-2 \pi n) \\
=-\sum_{n=-\infty}^{\infty} e^{2 \pi n i \beta} \delta(x, y-2 \pi n) .
\end{gathered}
$$

The result holds if we replace $1 / \mu_{m} \exp \left(\mu_{m}|x|+i(m+\beta) y\right)$ in $G(\mathbf{r})$ with $-1 / \mu_{m} \exp \left(-\mu_{m}|x|+i(m+\beta) y\right)$ for a finite number of integers $m$, because this amounts to adding a finite number of functions

$$
\psi_{m}=\frac{1}{\mu_{m}}\left(e^{-\mu_{m}|x|}+e^{\mu_{m}|x|}\right) e^{i(m+\beta) y}=\frac{1}{\mu_{m}}\left(e^{-\mu_{m} x}+e^{\mu_{m} x}\right) e^{i(m+\beta) y},
$$

which satisfy $\left(\nabla^{2}+\epsilon \omega^{2}\right) \psi_{m}=0$.

Finally, we note that the ellipticity of $\nabla^{2}+\epsilon \omega^{2}$ implies that any distribution solution on a domain must be a function of class $C^{\infty}$. Thus $G(\mathbf{r})$ is of class $C^{\infty}$ on $\mathbb{R}^{2} \backslash\{(0,2 \pi n): n \in \mathbb{Z}\}$.

For our purposes, we make the following choices of the sign of $\mu_{m}$. For real values of $\omega$ (such that $(m+\beta)^{2}-\epsilon \omega^{2} \neq 0$ for all integers $m$ ), we choose $\mu_{m}$ such that $G(\hat{\mathbf{r}})$ is a radiating Green's function. Thus, for the finite number of consecutive integers $m$ such that $(m+\beta)^{2}-\epsilon \omega^{2}<0$ (it is possible that there are no such values of $m$ ), we take $\mu_{m}$ to lie on the positive imaginary axis; these values of $m$ give the finite number of outwardly propagating modes. For all other values of $m$, we take $\mu_{m}<0$; these give the decaying modes.

In our investigations, we will consider continuous perturbations of $\omega$ into the lower-half complex plane, and we allow the values of $\mu_{m}$ to vary analytically with $\omega$. As $\omega$ attains a negative imaginary part, the finite number of values of $\mu_{m}$ that gave the propagating modes now attain a positive real part and therefore grow as $|x| \rightarrow \infty$. For all other values of $m, \mu_{m}$ attains a negative imaginary part.

Figure 2.1 shows the number of propagating modes for real values of $\beta$ and $\omega$. Pairs $(\beta, \omega)$ for which there are no propagating modes and perturbations of these in the imaginary $\omega$ direction admit no scattering (extended) EM fields in the strip $\mathcal{S}$.

We will make use of the set of Helmholtz fields which, to the right and left of the scatterer, are equal to a superposition of modes that build the Green's functions $G$ that we defined in Theorem 2.1.

Definition 2.2. We say that a function $\psi$ is in the class $\mathcal{E}(\beta, \omega, \epsilon)$ if its domain contains $\left\{(x, y):|x|>x_{*}\right\}$ for some $x_{*}>0$ and

$$
\psi(x, y)=\sum_{m=-\infty}^{\infty} A_{m}^{ \pm} \exp \left(\mu_{m}|x|+i(m+\beta) y\right) \quad \text { for } \quad \pm x>x_{*}
$$




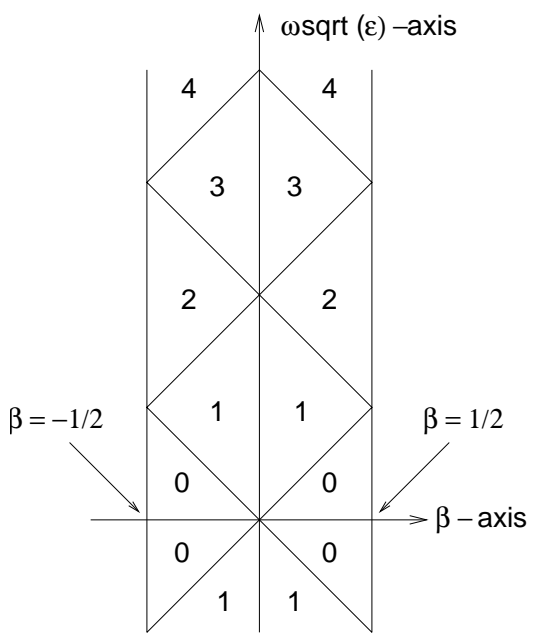

FIG. 2.1. The number of propagating modes for real values of $\beta$ and $\omega$. The pattern is repeated periodically in $\beta$, with period 1. For fixed $\beta$ and $\omega$, there is a propagating mode for each $m$ such that $|\sqrt{\epsilon} \omega|>|m+\beta|$.

for some complex numbers $A_{m}^{ \pm}$, where $\mu_{m}=\left((m+\beta)^{2}-\epsilon \omega^{2}\right)^{1 / 2}$ with the sign determination described above.

Remark. The role of the class $\mathcal{E}(\beta, \omega, \epsilon)$ is to continue analytically into the complex $\omega$-plane as $|x| \rightarrow \infty$ the standard outgoing radiation condition that applies when $\omega$ is real: $\mathcal{E}(\beta, \omega, \epsilon)$ contains the radiating fields when $\omega$ is real and their analytic continuations into the complex $\omega$-plane.

3. The boundary-integral projections. Let $\xi \in H^{1}(\partial D)$ and $\eta \in L^{2}(\partial D)$ be given $\left(H^{1}\right.$ is the linear space of functions on $\partial D$ with square-integrable arclength derivatives), and denote $\boldsymbol{\xi}=(\xi, \eta)^{t}$. For any point $\hat{\mathbf{r}}$ in the strip $\mathcal{S}$ exterior to $D$, define

$$
\psi(\hat{\mathbf{r}})=\int_{\partial D}\left(\frac{\partial G(\hat{\mathbf{r}}-\mathbf{r})}{\partial n(\mathbf{r})} \xi(\mathbf{r})-G(\hat{\mathbf{r}}-\mathbf{r}) \eta(\mathbf{r})\right) d s(\mathbf{r}) \quad(\hat{\mathbf{r}} \text { exterior to } D) .
$$

This field is an element of $\mathcal{E}(\beta, \omega, \epsilon)$. For $\hat{\mathbf{r}} \in D$, define

$$
\psi(\hat{\mathbf{r}})=\int_{\partial D}\left(-\frac{\partial G(\hat{\mathbf{r}}-\mathbf{r})}{\partial n(\mathbf{r})} \xi(\mathbf{r})+G(\hat{\mathbf{r}}-\mathbf{r}) \eta(\mathbf{r})\right) d s(\mathbf{r}) \quad(\hat{\mathbf{r}} \in D) .
$$

Both fields satisfy the Helmholtz equation (in the variable $\hat{\mathbf{r}}$ ) in their respective domains.

Let $\hat{\mathbf{r}}$ now be a point on $\partial D$, and consider the limits to $\hat{\mathbf{r}}$ of these exterior and interior fields and their normal derivatives:

$$
\begin{gathered}
\boldsymbol{\psi}_{e}(\hat{\mathbf{r}})=\left[\begin{array}{c}
\psi_{e}(\hat{\mathbf{r}}) \\
\partial_{n} \psi_{e}(\hat{\mathbf{r}})
\end{array}\right]:=\left[\begin{array}{c}
\lim _{h \rightarrow 0^{+}} \psi(\hat{\mathbf{r}}+h n(\hat{\mathbf{r}})) \\
\lim _{h \rightarrow 0^{+}} \frac{\partial \psi}{\partial n(\hat{\mathbf{r}})}(\hat{\mathbf{r}}+h n(\hat{\mathbf{r}}))
\end{array}\right], \\
\boldsymbol{\psi}_{i}(\hat{\mathbf{r}})=\left[\begin{array}{c}
\psi_{i}(\hat{\mathbf{r}}) \\
\partial_{n} \psi_{i}(\hat{\mathbf{r}})
\end{array}\right]:=\left[\begin{array}{c}
\lim _{h \rightarrow 0^{+}} \psi(\hat{\mathbf{r}}-h n(\hat{\mathbf{r}})) \\
\lim _{h \rightarrow 0^{+}} \frac{\partial \psi}{\partial n(\hat{\mathbf{r}})}(\hat{\mathbf{r}}-h n(\hat{\mathbf{r}}))
\end{array}\right] .
\end{gathered}
$$


These limits are again in $H^{1}(\partial D) \times L^{2}(\partial D)$. See the appendix for a more detailed discussion of these limits.

The limit of the first-order normal derivatives of the Green's function produces singular contributions according to the Plemelj formula, and we obtain

$$
\begin{aligned}
& \boldsymbol{\psi}_{e}=\frac{1}{2}(I+H) \boldsymbol{\xi}, \\
& \boldsymbol{\psi}_{i}=\frac{1}{2}(I-H) \boldsymbol{\xi},
\end{aligned}
$$

in which $H$ is an integral operator from $H^{1}(\partial D) \times L^{2}(\partial D)$ to itself defined by

$$
(H \boldsymbol{\xi})(\hat{\mathbf{r}})=2\left[\begin{array}{c}
\int_{\partial D}\left(\frac{\partial G(\hat{\mathbf{r}}-\mathbf{r})}{\partial n(\mathbf{r})} \xi(\mathbf{r})-G(\hat{\mathbf{r}}-\mathbf{r}) \eta(\mathbf{r})\right) d s(\mathbf{r}) \\
\lim _{h \rightarrow 0} \int_{\partial D} \frac{\partial G(\hat{\mathbf{r}}+h n(\hat{\mathbf{r}})-\mathbf{r})}{\partial n(\hat{\mathbf{r}}) \partial n(\mathbf{r})} \xi(\mathbf{r}) d s(\mathbf{r})-\int_{\partial D} \frac{\partial G(\hat{\mathbf{r}}-\mathbf{r})}{\partial n(\hat{\mathbf{r}})} \eta(\mathbf{r}) d s(\mathbf{r})
\end{array}\right] .
$$

$H$ has the form

$$
H\left[\begin{array}{l}
\xi \\
\eta
\end{array}\right]=2\left[\begin{array}{cc}
K & -J \\
L & -K^{\prime}
\end{array}\right]\left[\begin{array}{l}
\xi \\
\eta
\end{array}\right],
$$

in which the entries of the matrix are integral operators on $\partial D$. The integral kernels of $J, K$, and $K^{\prime}$ are $G(\hat{\mathbf{r}}-\mathbf{r}), \partial G(\hat{\mathbf{r}}-\mathbf{r}) / \partial n(\mathbf{r})$, and $\partial G(\hat{\mathbf{r}}-\mathbf{r}) / \partial n(\hat{\mathbf{r}})$, respectively. By integration by parts and using the identity

$$
\frac{\partial^{2} G(\hat{\mathbf{r}}-\mathbf{r})}{\partial n(\hat{\mathbf{r}}) \partial n(\mathbf{r})}+\frac{\partial^{2} G(\hat{\mathbf{r}}-\mathbf{r})}{\partial s(\hat{\mathbf{r}}) \partial s(\mathbf{r})}=-n(\hat{\mathbf{r}}) \cdot n(\mathbf{r})\left(\partial_{x}^{2}+\partial_{y}^{2}\right) G(\hat{\mathbf{r}}-\mathbf{r}),
$$

one can show that the integral kernel of $L$ is

$$
n(\hat{\mathbf{r}}) \cdot n(\mathbf{r}) \epsilon \omega^{2} G(\hat{\mathbf{r}}-\mathbf{r})+\frac{\partial G(\hat{\mathbf{r}}-\mathbf{r})}{\partial s(\hat{\mathbf{r}})} \frac{d}{d s},
$$

where $s$ is the arclength parameter and $\partial G / \partial s$ is a principal-value kernel (see the proof of Theorem 2.1 in [2]).

THEOREM 3.1. $H$ is a bounded linear operator from $H^{1}(\partial D) \times L^{2}(\partial D)$ into itself, the operators

$$
\begin{aligned}
& P_{e}=\frac{1}{2}(I+H), \\
& P_{i}=\frac{1}{2}(I-H)
\end{aligned}
$$

are complementary projections, and

$$
H^{2}=I \text {. }
$$

Proof. Let us consider a solution $\psi$ of $\nabla^{2} \psi+\epsilon \omega^{2} \psi=0$ defined in $D$ that has a continuous extension to $\bar{D}$ whose restriction to $\partial D$ is in $H^{1}(\partial D)$ and such that $\lim _{h \rightarrow 0^{-}} \partial \psi / d n(\mathbf{r})(\mathbf{r}+h n(\mathbf{r}))$ exists and belongs to $L^{2}(\partial D)$. Then Green's identity holds: For $\hat{\mathbf{r}} \in D$,

$$
\psi(\hat{\mathbf{r}})=\int_{\partial D}\left(-\frac{\partial G(\hat{\mathbf{r}}-\mathbf{r})}{\partial n(\mathbf{r})} \psi(\mathbf{r})+G(\hat{\mathbf{r}}-\mathbf{r}) \frac{\partial \psi(\mathbf{r})}{\partial n(\mathbf{r})}\right) d s(\mathbf{r}) .
$$


Green's identity says that (3.2) holds if we put $\psi$ and $\partial_{n} \psi$ in place of $\xi$ and $\eta$, and therefore

$$
P_{i}^{2}(\boldsymbol{\xi})=P_{i}\left(\boldsymbol{\psi}_{\boldsymbol{i}}\right)=\boldsymbol{\psi}_{\boldsymbol{i}}=P_{i}(\boldsymbol{\xi})
$$

and thus $P_{i}^{2}=P_{i}$.

Now let a solution $\psi$ of $\nabla^{2} \psi+\epsilon \omega^{2} \psi=0$ such that $\psi \in \mathcal{E}(\beta, \omega, \epsilon)$ be defined in $\mathcal{S} \backslash \bar{D}$, and suppose that $\psi$ has a continuous extension to $\bar{D}$ whose restriction to $\partial D$ is in $H^{1}(\partial D)$ and such that $\lim _{h \rightarrow 0^{-}} \partial \psi / d n(\mathbf{r})(\mathbf{r}+h n(\mathbf{r}))$ exists and belongs to $L^{2}(\partial D)$. Again, Green's identity holds: For $\hat{\mathbf{r}} \in \mathcal{S} \backslash \bar{D}$,

$$
\psi(\hat{\mathbf{r}})=\int_{\partial D}\left(\frac{\partial G(\hat{\mathbf{r}}-\mathbf{r})}{\partial n(\mathbf{r})} \psi(\mathbf{r})-G(\hat{\mathbf{r}}-\mathbf{r}) \frac{\partial \psi(\mathbf{r})}{\partial n(\mathbf{r})}\right) d s(\mathbf{r}) .
$$

The contribution from the upper and lower sides of the strip $\mathcal{S}$ cancel because of the pseudoperiodicity of $\psi$ and $G^{\epsilon}$. Straightforward calculation shows that the contributions from vertical line segments truncating the strip on the left and right vanish identically as the points of truncation tend to infinity, because both $G$ and $\psi$ are in $\mathcal{E}(\beta, \omega, \epsilon)$. Again, we find that $P_{e}^{2}=P_{e}$ by putting $\psi$ and $\partial_{n} \psi$ in place of $\xi$ and $\eta$ in $(3.1)$.

It is straightforward to calculate that $H^{2}=I$.

The operators in Theorem 3.1 are boundary-integral projections of Calderón's type. See Calderón [7], Seeley [8], and Ryaben'kii [9]. Nédélec [10] derives the Calderón's projections for the full harmonic Maxwell equations for a bounded domain in $\mathbb{R}^{3}$.

4. The Fredholm system of boundary-integral equations. We describe the EM scattering by a photonic crystal slab in terms of the decomposition given by the boundary-integral projections. We have seen that any pair of functions $(\xi, \eta)^{t}=\boldsymbol{\xi}$ in $H^{1}(\partial D) \times L^{2}(\partial D)$ can be expressed uniquely as the sum of the limiting values to $\partial D$ of an interior Helmholtz field and its normal derivative and an exterior Helmholtz field in $\mathcal{E}(\beta, \omega, \epsilon)$ and its normal derivative.

DeFINITION 4.1. An exterior-source field $\boldsymbol{\xi}=\phi_{i}^{\epsilon_{0}}$ has zero exterior component in the above decomposition over the exterior medium $\left(\epsilon=\epsilon_{0}\right)$; that is,

$$
P_{e}^{\epsilon_{0}} \phi_{i}^{\epsilon_{0}}=0, \quad P_{i}^{\epsilon_{0}} \boldsymbol{\phi}_{i}^{\epsilon_{0}}=\boldsymbol{\phi}_{i}^{\epsilon_{0}} .
$$

An interior-source field $\boldsymbol{\xi}=\boldsymbol{\phi}_{e}^{\epsilon_{1}}$ has zero interior component in the above decomposition over the interior medium $\left(\epsilon=\epsilon_{1}\right)$; that is,

$$
P_{i}^{\epsilon_{1}} \phi_{e}^{\epsilon_{1}}=0, \quad P_{e}^{\epsilon_{1}} \phi_{e}^{\epsilon_{1}}=\phi_{e}^{\epsilon_{1}} .
$$

In other words, when the sources are in the exterior, the source field extends from $\partial D$ to a Helmholtz field over the medium $\epsilon_{0}$ in the interior. Similarly, when the sources are in the interior, the source field extends from $\partial D$ to a Helmholtz field of class $\mathcal{E}\left(\beta, \omega, \epsilon_{1}\right)$ over the medium $\epsilon_{1}$ in the exterior.

Remark. In our numerical calculations of transmission in section 7, we use planewave source fields from the left at real frequencies $\omega$; that is,

$$
\psi_{i}^{\epsilon_{0}}=\exp \left(i \sqrt{\epsilon_{0} \omega^{2}-(m+\beta)^{2}} x+i(m+\beta) y\right), \quad \psi_{e}^{\epsilon_{1}}=0 .
$$

A solution to the scattering problem has total exterior and interior fields $\psi_{\text {ext }}$ and $\psi_{\text {int }}$, with traces (field and normal derivative) on $\partial D$ given by

$$
\boldsymbol{\psi}_{\mathrm{ext}}=\boldsymbol{\psi}_{e}^{\epsilon_{0}}+\boldsymbol{\phi}_{i}^{\epsilon_{0}}, \quad \boldsymbol{\psi}_{\mathrm{int}}=\boldsymbol{\psi}_{i}^{\epsilon_{1}}+\boldsymbol{\phi}_{e}^{\epsilon_{1}},
$$


where $\boldsymbol{\psi}_{i}^{\epsilon_{1}}$ and $\boldsymbol{\psi}_{e}^{\epsilon_{0}}$ are the traces of the interior and exterior scattered fields. The field $\boldsymbol{\psi}_{i}^{\epsilon_{1}}$ extends to a Helmholtz field in the interior, and $\boldsymbol{\psi}_{e}^{\epsilon_{0}}$ extends to a Helmholtz field of class $\mathcal{E}\left(\beta, \omega, \epsilon_{0}\right)$ in the exterior. When $\omega$ is real, the exterior extension of $\boldsymbol{\psi}_{e}^{\epsilon_{0}}$ either decays or satisfies the outward radiation condition as $|x| \rightarrow \infty$.

The matching conditions at the interface of the two media are given by

$$
\left[\begin{array}{c}
\psi_{\text {int }}(\hat{\mathbf{r}}) \\
\partial_{n} \psi_{\text {int }}(\hat{\mathbf{r}})
\end{array}\right]=\left[\begin{array}{ll}
1 & 0 \\
0 & \nu
\end{array}\right]\left[\begin{array}{c}
\psi_{\text {ext }}(\hat{\mathbf{r}}) \\
\partial_{n} \psi_{\text {ext }}(\hat{\mathbf{r}})
\end{array}\right] .
$$

We write the matching conditions in short form by defining $\Gamma=\left[\begin{array}{ll}1 & 0 \\ 0 & \nu\end{array}\right]$ and $\boldsymbol{\psi}=$ $\left[\begin{array}{c}\psi_{\text {ext }}(\hat{\mathbf{r}}) \\ \partial_{n} \psi_{\text {ext }}(\hat{\mathbf{r}})\end{array}\right]$ on $\partial D$ and inserting them into (4.1) to obtain

$$
\boldsymbol{\psi}=\boldsymbol{\psi}_{e}^{\epsilon_{0}}+\boldsymbol{\phi}_{i}^{\epsilon_{0}}, \quad \Gamma \boldsymbol{\psi}=\boldsymbol{\psi}_{i}^{\epsilon_{1}}+\boldsymbol{\phi}_{e}^{\epsilon_{1}} .
$$

We apply the projections $P_{i}^{\epsilon_{0}}$ and $P_{e}^{\epsilon_{1}}$ to the two equations, respectively,

$$
P_{i}^{\epsilon_{0}} \boldsymbol{\psi}=\phi_{i}^{\epsilon_{0}} \quad \text { and } \quad P_{e}^{\epsilon_{1}} \Gamma \boldsymbol{\psi}=\boldsymbol{\phi}_{e}^{\epsilon_{1}} .
$$

Definition 4.2. By the scattering problem at $(\beta, \omega)$, we mean the system of equations (4.4) with the source fields (see Definition 4.1) in the right-hand sides belonging to the space $H^{1}(\partial D) \times L^{2}(\partial D)$. A scattering state or scattering field is a Helmholtz field whose trace on $\partial D$ is a solution of the system with a nonzero source. If there exists a nontrivial solution $\boldsymbol{\psi}$ in the absence of sources (zero right-hand sides), we call the frequency $\omega$ a resonant frequency (for the wave number $\beta$ ). The corresponding Helmholtz field is necessarily in $\mathcal{E}\left(\beta, \omega, \epsilon_{0}\right)$. If the field decays as $|x| \rightarrow \infty$, we call it a bound state.

Remarks. 1. Our definition of scattering state analytically continues the traditional scattering states at real frequencies into the complex $\omega$-plane. This is possible by our analytic continuation of the condition that defines the notion of an outgoing radiating field (see the Remark after Definition 2.2). A scattering state is an analytic function of $\omega$, and a resonant frequency is a singularity of this function.

2. We say that a scattering field at a nonresonant real frequency near a resonant frequency exhibits resonant behavior if it exhibits amplitudes in the crystal structure that are large compared to the source amplitude.

3. We will need to consider only frequencies $\omega$ such that $\Re(\omega)>0$. We will prove in Theorems 5.1 and 5.2 that, in this case, a dielectric photonic crystal slab supports only Helmholtz fields with $\Im(\omega) \leq 0$ and that such a field decays as $|x| \rightarrow \infty$ if $\Im(\omega)=0$, and is unbounded if $\Im(\omega)<0$.

We add the two equations (4.4) to obtain

$$
\left(P_{i}^{\epsilon_{0}}+P_{e}^{\epsilon_{1}} \Gamma\right) \boldsymbol{\psi}=\phi_{i}^{\epsilon_{0}}+\phi_{e}^{\epsilon_{1}} .
$$

Inserting the expressions

$$
P_{i}^{\epsilon_{0}}=\frac{1}{2}\left(I+H^{\epsilon_{0}}\right), \quad P_{e}^{\epsilon_{1}}=\frac{1}{2}\left(I-H^{\epsilon_{1}}\right)
$$

from Theorem 3.1, and letting $\phi=\phi_{i}^{\epsilon_{0}}+\phi_{e}^{\epsilon_{1}}$ represent the total source field, we rewrite (4.5) as

$$
\left[\frac{1}{2}(I+\Gamma)+\frac{1}{2}\left(H^{\epsilon_{0}}-H^{\epsilon_{1}} \Gamma\right)\right] \boldsymbol{\psi}=\boldsymbol{\phi} .
$$


Finally, we insert the expression (3.3) for $H^{\epsilon_{0}}$ and $H^{\epsilon_{1}}$ to obtain the following expanded version of (4.5) (recall $\boldsymbol{\psi}=\boldsymbol{\psi}_{\text {ext }}$ ):

$$
\begin{aligned}
\psi_{\text {ext }}(\hat{\mathbf{r}})+\int_{\partial D}\left[\frac{\partial\left(G^{\epsilon_{1}}-G^{\epsilon_{0}}\right)(\hat{\mathbf{r}}-\mathbf{r})}{\partial n(\mathbf{r})} \psi_{\mathrm{ext}}(\mathbf{r})\right. \\
\left.\left.\left.-\left(\nu G^{\epsilon_{1}}-G^{\epsilon_{0}}\right)(\hat{\mathbf{r}}-\mathbf{r}) \frac{\partial \psi_{\mathrm{ext}}}{\partial n(\mathbf{r})}\right] \mathbf{r}\right)\right] d s(\mathbf{r})=\phi(\hat{\mathbf{r}}),
\end{aligned}
$$

$$
\frac{1+\nu}{2} \frac{\partial \psi_{\text {ext }}}{\partial n(\hat{\mathbf{r}})}(\hat{\mathbf{r}})+\int_{\partial D}\left[\frac{\partial^{2}\left(G^{\epsilon_{1}}-G^{\epsilon_{0}}\right)(\hat{\mathbf{r}}-\mathbf{r})}{\partial n(\hat{\mathbf{r}}) \partial n(\mathbf{r})} \psi_{\mathrm{ext}}(\mathbf{r})\right.
$$

$$
\left.-\frac{\partial\left(\nu G^{\epsilon_{1}}-G^{\epsilon_{0}}\right)(\hat{\mathbf{r}}-\mathbf{r})}{\partial n(\hat{\mathbf{r}})} \frac{\partial \psi_{\text {ext }}}{\partial n(\mathbf{r})}(\mathbf{r})\right] d s(\mathbf{r})=\frac{\partial \phi}{\partial n(\hat{\mathbf{r}})}(\hat{\mathbf{r}}) .
$$

This is a Fredholm equation of the second kind; cancellation in the difference of the Green's functions reduces the leading singularities of all the kernels to at most logarithmic, making the corresponding operators of Hilbert-Schmidt class in $L^{2}(\partial D) \times$ $L^{2}(\partial D)$. Equation (4.6) can thus be solved for $\boldsymbol{\psi}$ in $L^{2}(\partial D) \times L^{2}(\partial D)$ whenever the nullspace is trivial. The solution $\boldsymbol{\psi}$ necessarily belongs to $H^{1}(\partial D) \times L^{2}(\partial D)$; indeed, (4.6) can be written as $\boldsymbol{\psi}=M \boldsymbol{\psi}+2(I+\Gamma)^{-1} \boldsymbol{\phi}$, where $M$ consists of kernels with at most logarithmic singularities, and is thus bounded from $L^{2}(\partial D) \times L^{2}(\partial D)$ to $H^{1}(\partial D) \times H^{1}(\partial D)$. In fact, $M$ is bounded from $H^{s}(\partial D) \times H^{s}(\partial D)$ to $H^{s+1}(\partial D) \times$ $H^{s+1}(\partial D)$, and so $\boldsymbol{\psi}$ is $C^{\infty}$ if $\phi=0$, i.e., if $\boldsymbol{\psi}$ is a nullfield. In summary, if the source field $\boldsymbol{\phi}$ belongs to $H^{1}(\partial D) \times L^{2}(\partial D)$, then so does the field $\boldsymbol{\psi}$.

Boundary integral equations of the second kind for EM fields have also been derived by Müller [11], Colton and Kress [12], [13], and Nédélec [10].

THEOREM 4.3. If the system

$$
P_{e}^{\epsilon_{0}} \boldsymbol{f}=0, \quad P_{i}^{\epsilon_{1}} \boldsymbol{f}=0
$$

has only the trivial solution, then the scattering problem (4.4) is equivalent to the Fredholm system (4.5) (equivalently, (4.6)).

Proof. Given (4.5), we may write

$$
P_{i}^{\epsilon_{0}} \boldsymbol{\psi}=\boldsymbol{\phi}_{i}^{\epsilon_{0}}+\boldsymbol{f}, \quad P_{e}^{\epsilon_{1}} \Gamma \boldsymbol{\psi}=\boldsymbol{\phi}_{e}^{\epsilon_{1}}-\boldsymbol{f} .
$$

Applying $P_{e}^{\epsilon_{0}}$ to the first relation and $P_{i}^{\epsilon_{1}}$ to the second relation, we obtain

$$
P_{e}^{\epsilon_{0}} \boldsymbol{f}=0, \quad P_{i}^{\epsilon_{1}} \boldsymbol{f}=0 .
$$

These equations imply $\boldsymbol{f}=0$ by the hypothesis of the theorem.

A comparison of the conditions of the theorem with (4.4) allows us to reformulate the theorem in a more physical way, as follows.

THEOREM 4.4 (reformulated Theorem (4.3)). If the inverse dielectric structure (physics terminology meaning the original geometry with the two dielectric materials interchanged) does not support an electrically polarized field, then our scattering problem (4.4) is equivalent to the Fredholm system (4.5) (equivalently, (4.6)).

5. Resonant frequencies and bound states. We have seen that the dielectric structure supports a nonzero source-free electromagnetic field $\psi$ at $(\beta, \omega)$ if and only if the field satisfies

$$
P_{i}^{\epsilon_{0}} \boldsymbol{\psi}=0 \quad \text { and } \quad P_{e}^{\epsilon_{1}} \Gamma \boldsymbol{\psi}=0
$$


where $\boldsymbol{\psi}(\mathbf{r})=\left(\lim _{h \rightarrow 0} \psi(\mathbf{r}+h n(\mathbf{r})), \lim _{h \rightarrow 0} \partial_{n} \psi(\mathbf{r}+h n(\mathbf{r}))\right)^{t}$ on $\partial D$. This implies that

$$
\left(P_{i}^{\epsilon_{0}}+P_{e}^{\epsilon_{1}} \Gamma\right) \boldsymbol{\psi}=0
$$

so that $\left(P_{i}^{\epsilon_{0}}+P_{e}^{\epsilon_{1}} \Gamma\right)$ has a nontrivial nullspace at the resonant frequency $\omega$ for wave number $\beta$.

We now present a theorem that provides conditions for the existence of resonance frequencies.

THEOREM 5.1. Let the frequency $\omega$ and a real value of $\beta$ be given, and assume that $(m+\beta)^{2}-\epsilon \omega^{2} \neq 0$ for all integers $m$. If $P_{i}^{\epsilon_{0}}+P_{e}^{\epsilon_{1}} \Gamma$ has a nontrivial nullspace, then at least one of the following holds:

(1) $\omega$ is a resonant frequency for the structure, with interior dielectric coefficient $\epsilon_{1}$ and exterior coefficient $\epsilon_{0}$ for the wave number $\beta$, according to whether $\nu=1$ or $\nu=\epsilon_{1} / \epsilon_{0}$ in $\Gamma$, or

(2) $\omega$ is a resonant frequency for the inverse structure, with $\epsilon_{1}$ and $\epsilon_{0}$ switched, for the wave number $\beta$.

If $\omega^{2}$ is real, then the corresponding source-free Helmholtz field $\psi$ in $\mathcal{E}\left(\beta, \omega, \epsilon_{0}\right)$ or $\mathcal{E}\left(\beta, \omega, \epsilon_{1}\right)$ decays to zero as $|x| \rightarrow \infty$ and is therefore an $x$-localized field (a bound state in the strip $\mathcal{S})$. Otherwise, $\Im\left(\omega^{2}\right)<0$ and $\psi$ becomes unbounded as $|x| \rightarrow \infty$.

Remarks. 1. In our numerical studies, we will be interested only in the case in which $\Re(\omega)>0$ and $\Im(\omega)$ is small and negative. Assuming $\Re(\omega)>0$, the condition $\Im\left(\omega^{2}\right)<0$ is equivalent to $\Im(\omega)<0$.

2. Suppose that the pair $\left(\beta_{0}, \omega_{0}\right)$ with $\omega_{0}>0$ admits a bound state and that the Green's function has no propagating modes (see Figure 2.1). Then, for $(\beta, \omega)$ in a neighborhood of $\left(\beta_{0}, \omega_{0}\right)$, the Green's function has only decaying modes, so that all functions in $\mathcal{E}\left(\beta, \omega, \epsilon_{0}\right)$ are decaying. This implies that, in a vicinity of $\beta_{0}$ (we always assume $\beta$ is real), a relation $\omega=W(\beta)$ describing resonant frequencies must be real-valued and must therefore be a dispersion relation for bound states.

Proof. We assume that (5.2) holds for some nonzero $\psi$. Theorem 4.3, with both source fields in (4.4) taken to be zero, implies that equations (5.1) hold (giving statement (1) in Theorem 5.1) or that there exists a nonzero $\boldsymbol{f}$ such that $P_{e}^{\epsilon_{0}} \boldsymbol{f}=0$ and $P_{i}^{\epsilon_{1}} \boldsymbol{f}=0$ (giving statement (2) in Theorem 5.1).

We defer the proof of the condition on $\omega^{2}$ and the behavior of the fields as $|x| \rightarrow \infty$ to the proof of Theorem 5.2.

THEOREM 5.2. Suppose that $\psi \in \mathcal{E}\left(\beta, \omega, \epsilon_{0}\right)$ is pseudoperiodic in $y$, is not identically zero, and satisfies $\nabla^{2} \psi+\epsilon \omega^{2} \psi=0$ in $\mathcal{S} \backslash \partial D$ (where $\epsilon=\epsilon_{1}$ in $D$ and $\epsilon_{0}$ otherwise) and the matching conditions (4.2) on $\partial D$. Then $\Im\left(\omega^{2}\right) \leq 0$. In addition, $|\psi| \rightarrow 0$ as $|x| \rightarrow \infty$ if and only if $\omega^{2}$ is real-valued.

Proof. Let $T$ denote the finite strip $\left\{(x, y):-x_{0} \leq x \leq x_{0}, 0 \leq y \leq 2 \pi\right\}$, where $x_{0}>x_{*}>0$ and $x_{*}$ is given in Definition 2.2, and let $\partial T$ be its boundary with outward-pointing normal vector $n$. We also take $n$ pointing outward on $\partial D$. The divergence theorem gives

$$
\int_{\partial T} \bar{\psi} \partial_{n} \psi d s+\int_{\partial D}\left(\bar{\psi}_{\text {int }} \partial_{n} \psi_{\text {int }}-\bar{\psi}_{\text {ext }} \partial_{n} \psi_{\text {ext }}\right) d s=\iint_{T}\left(\nabla \bar{\psi} \cdot \nabla \psi+\bar{\psi} \nabla^{2} \psi\right) d A .
$$

Using the relation $\bar{\psi} \partial_{n} \psi=\bar{\psi} \partial_{n} \tilde{\psi}+i \beta n_{y}|\tilde{\psi}|^{2}\left(n_{y}\right.$ is the $y$-component of the normal vector $n$ ), we see that the integrals over the top and bottom parts of $\partial T$ cancel, and the integral over $\partial T$ becomes an integral over $\Gamma_{L} \cup \Gamma_{R}$, where $\Gamma_{L}$ and $\Gamma_{R}$ are the left 
and right sides of $\partial T$. On the right-hand side, we use the Helmholtz equation. We now have

$$
\int_{\Gamma_{L} \cup \Gamma_{R}} \bar{\psi} \partial_{n} \psi d s+\int_{\partial D}\left(\bar{\psi}_{\text {int }} \partial_{n} \psi_{\text {int }}-\bar{\psi}_{\text {ext }} \partial_{n} \psi_{\text {ext }}\right) d s=\iint_{T}\left(|\nabla \psi|^{2}-\epsilon \omega^{2}|\psi|^{2}\right) d A .
$$

Using the conjugate equation for the interior of $D, \nabla^{2} \bar{\psi}_{\text {int }}+\epsilon_{1} \bar{\omega}^{2} \bar{\psi}_{\text {int }}=0$, we compute

$$
\begin{aligned}
& -\iint_{D}\left|\psi_{\text {int }}\right|^{2}\left(\epsilon_{1} \omega^{2}-\epsilon_{1} \bar{\omega}^{2}\right) d A=-\iint_{D}\left(\bar{\psi}_{\text {int }} \epsilon_{1} \omega^{2} \psi_{\text {int }}-\psi_{\text {int }} \epsilon_{1} \bar{\omega}^{2} \bar{\psi}_{\text {int }}\right) d A \\
& =\iint_{D}\left(\bar{\psi}_{\text {int }} \nabla^{2} \psi_{\text {int }}-\psi_{\text {int }} \nabla^{2} \bar{\psi}_{\text {int }}\right) d A=\int_{\partial D}\left(\bar{\psi}_{\text {int }} \partial_{n} \psi_{\text {int }}-\psi_{\text {int }} \partial_{n} \bar{\psi}_{\text {int }}\right) d s .
\end{aligned}
$$

Therefore,

$$
\Im \int_{\partial D} \bar{\psi}_{\text {int }} \partial_{n} \psi_{\text {int }} d s=-\epsilon_{1} \Im\left(\omega^{2}\right) \iint_{D}\left|\psi_{\text {int }}\right|^{2} d A .
$$

Using this and the matching conditions $\psi_{\text {int }}=\psi_{\text {ext }}$ and $\partial_{n} \psi_{\text {int }}=\nu \partial_{n} \psi_{\text {ext }}$ on $\partial D$, we can write the imaginary part of (5.3):

$$
\Im \int_{\Gamma_{L} \cup \Gamma_{R}} \bar{\psi} \partial_{n} \psi d s-\epsilon_{1}\left(1-\nu^{-1}\right) \Im\left(\omega^{2}\right) \iint_{D}|\psi|^{2} d A=-\Im\left(\omega^{2}\right) \iint_{T} \epsilon|\psi|^{2} d A .
$$

By Definition 2.2, since $\psi \in \mathcal{E}\left(\beta, \omega, \epsilon_{0}\right)$, there exist complex numbers $A_{m}^{ \pm}$such that

$$
\psi(x, y)=\sum_{m=-\infty}^{\infty} A_{m}^{ \pm} \exp \left(\mu_{m}|x|+i(m+\beta) y\right) \quad \text { for } \quad \pm x>x_{0} .
$$

Straightforward computation yields

$$
\int_{\Gamma_{L} \cup \Gamma_{R}} \bar{\psi} \partial_{n} \psi d s=2 \pi \sum_{m=-\infty}^{\infty} \mu_{m}\left(\left|A_{m}^{-}\right|^{2}+\left|A_{m}^{+}\right|^{2}\right) e^{2 \Re\left(\mu_{m}\right)\left|x_{0}\right|},
$$

and, after splitting the right-hand side of (5.4) into an interior and an exterior integral, we obtain

$$
\begin{aligned}
2 \pi \sum_{m=-\infty}^{\infty} \Im\left(\mu_{m}\right) & \left(\left|A_{m}^{-}\right|^{2}+\left|A_{m}^{+}\right|^{2}\right) e^{2 \Re\left(\mu_{m}\right)\left|x_{0}\right|} \\
& =-\Im\left(\omega^{2}\right)\left(\epsilon_{1} \nu^{-1} \iint_{D}|\psi|^{2} d A+\epsilon_{0} \iint_{T \backslash D}|\psi|^{2} d A\right) .
\end{aligned}
$$

If $\Im\left(\omega^{2}\right)>0$, all modes are decaying in $x\left(\Re\left(\mu_{m}\right)<0\right.$ for all $\left.m\right)$, and we obtain a contradiction by letting $x_{0}$ tend to $\infty$; therefore, $\Im\left(\omega^{2}\right) \leq 0$. If $\Im\left(\omega^{2}\right)=0$, then $\Im\left(\mu_{m}\right)>0$ for all propagating modes and $\Im\left(\mu_{m}\right)=0$ for all decaying modes; therefore, $A_{m}^{ \pm}=0$ for all propagating modes, so that $|\psi| \rightarrow 0$ as $|x| \rightarrow \infty$. Conversely, if $|\psi| \rightarrow 0$ as $|x| \rightarrow \infty$, then $A_{m}^{ \pm}=0$ for all nondecaying modes (those for which $\Re\left(\mu_{m}\right) \geq 0$ ), and thus the left-hand side of 5.5 decays exponentially as $x_{0} \rightarrow \infty$. Letting $x_{0}$ tend to $\infty$ shows that $\Im\left(\omega^{2}\right)=0$.

Remarks. 1. The quantity $\Im \int_{\Gamma} \bar{\psi} \partial_{n} \psi d s$ appearing in the proof of Theorem 5.2 is the time-averaged energy flow carried by $\psi$ through $\Gamma$.

2. If $\Im\left(\omega^{2}\right)<0$, then $\psi$ does not decay as $|x| \rightarrow \infty$, and thus by the definition of $\mathcal{E}(\beta, \omega, \epsilon), \psi$ becomes unbounded as $|x| \rightarrow \infty$, and this completes the proof of Theorem 5.1. 
6. Dispersion relations. We define a dispersion relation for a photonic crystal slab to be a multivalued function $\omega=W(\beta)$ describing pairs $(\beta, \omega)$ for which $\omega$ is a resonant frequency for wave number $\beta$. This means that the pair (5.1) is satisfied for a nonzero field $\boldsymbol{\psi}(\mathbf{r})=\left(\lim _{h \rightarrow 0} \psi(\mathbf{r}+h n(\mathbf{r})), \lim _{h \rightarrow 0} \partial_{n} \psi(\mathbf{r}+h n(\mathbf{r}))\right)^{t}$ on $\partial D$, where $\psi$ is a pseudoperiodic source-free Helmholtz field in the class $\mathcal{E}\left(\beta, \omega, \epsilon_{0}\right)$. If we are able to eliminate the second alternative in Theorem 5.1, then the single equation (5.2) is sufficient for defining a dispersion relation, as it would then imply the pair (5.1). In the numerical examples below, we are able computationally to eliminate the second alternative.

Because our operator is of the Hilbert-Schmidt class from $L^{2}(\partial D) \times L^{2}(\partial D)$, its determinant is defined, and it depends analytically on $\beta$ and $\omega$. Thus, a necessary condition for the pair $(\beta, \omega)$ to support a source-free field in the given crystal is

$$
D(\beta, \omega):=\operatorname{det}\left(P_{i}^{\epsilon_{0}}+P_{e}^{\epsilon_{1}} \Gamma\right)=0 .
$$

The dispersion relation is therefore given by branches of $D(\beta, \omega)=0$. Whether this condition is also sufficient depends on the inverse dielectric structure. Indeed, we know from Theorem 5.1 that if the inverse structure does not support an electrically polarized source-free field, then (6.1) does define a dispersion relation for fields in the original structure.

In practice, we obtain dispersion relations by computing the curves $\lambda(\beta, \omega)=0$ numerically, where $\lambda$ is an eigenvalue of $P_{i}^{\epsilon_{0}}+P_{e}^{\epsilon_{1}} \Gamma$. Suppose that zero is a simple eigenvalue at the pair $\left(\beta_{0}, \omega_{0}\right)$. Then the smallest eigenvalue $\lambda$ is an analytic function of $\beta$ and $\omega$ in a neighborhood of $\left(\beta_{0}, \omega_{0}\right)$, say $\lambda=\lambda(\beta, \omega)$. Suppose also that $\lambda\left(\beta_{0}, \omega\right) \not \equiv$ 0 near $\omega_{0}$. By the Weierstraß preparation theorem, there exists an integer $n \geq 1$ such that

$$
\lambda(\beta, \omega)=h(\beta, \omega)\left(\omega^{n}+W_{n-1}(\beta) \omega^{n-1}+\cdots+W_{1}(\beta) \omega+W_{0}(\beta)\right)
$$

near $\left(\beta_{0}, \omega_{0}\right)$, where $h$ and $W_{i}(i=0, \ldots, n-1)$ are analytic functions and $h \neq 0$ near $\left(\beta_{0}, \omega_{0}\right)$. Thus, $\lambda=0$ is equivalent to $\omega^{n}+W_{n-1}(\beta) \omega^{n-1}+\cdots+W_{1}(\beta) \omega+W_{0}(\beta)=0$. Let us consider the case in which $n=1$ (this is when $\partial \lambda / \partial \omega \neq 0$ near $\left(\beta_{0}, \omega_{0}\right)$ ). Then we have a relation

$$
\omega=W(\beta)
$$

that describes the locus of $(\beta, \omega)$-pairs for which $\lambda=0$. For real values of $\beta$ near $\beta_{0}$, the curve $\omega=W(\beta)$ in the complex $\omega$-plane gives a dispersion relation; it is periodic in $\beta$ with period 1 .

We made two assumptions in the preceding paragraph: that zero is a simple eigenvalue at $\left(\beta_{0}, \omega_{0}\right)$, and that $\partial \lambda / \partial \omega \neq 0$ there. Numerical calculations show that both assumptions are true generically, giving rise to dispersion relations for simple eigenvalues. When two branches cross, as in Figure 7.3(1a) and (2a) below, we see an eigenvalue of multiplicity 2 . We have not encountered a situation in which there is a simple eigenvalue at some point $\left(\beta_{0}, \omega_{0}\right)$ and $\partial \lambda / \partial \omega=0$ there, that is, $\partial^{k} \lambda / \partial \omega^{k}=0$ for $k<n$ and $\partial^{n} \lambda / \partial \omega^{n} \neq 0$ for some $n>1$ in (6.2). In this situation, (6.2) shows that there would exist a dispersion relation defined by an equation that is algebraic in $\omega$ and may have several branches emanating from $\left(\beta_{0}, \omega_{0}\right)$.

If $\omega=W(\beta)$ lies on the real $\omega$-axis over a range of $\beta$-values, then we have a dispersion relation for $x$-localized Helmholtz fields $\psi$ in the crystal (bound states in the strip $\mathcal{S}$ ). The complex time-dependent electric or magnetic fields associated with 
$\psi$ are $\psi(x, y) e^{-i \omega t}=\tilde{\psi}(x, y) e^{i(\beta y-\omega t)}$ (with $\tilde{\psi}$ periodic in $y$ ), which are Bloch waves traveling along the photonic crystal slab. If $\omega_{0}=W\left(\beta_{0}\right)$ is real but the dispersion relation goes into the lower-half $\omega$-plane for $\beta \neq \beta_{0}$, then there exists a bound state just for the isolated pair $\left(\beta_{0}, \omega_{0}\right)$, and nearby pairs $(\beta, W(\beta))$ are complex resonant frequencies. We demonstrate numerically below that both situations do occur.

One of the main phenomena observed in this study is that complex resonant frequencies are linked to resonant scattering behavior and transmission anomalies at nearby real frequencies. See also [5].

7. Numerical studies: Bound states, surface waves, and resonances. In this section we present three examples that illustrate the connections between complex dispersion relations, bound states and resonant frequencies, transmission anomalies, and resonant behavior of real frequencies. In particular, we calculate a dispersion relation for surface waves at bandgap frequencies on a thick structure approximating a semi-infinite crystal, and we provide a mathematical context for understanding resonant phenomena produced by a channel defect in a crystal slab.

We calculate the dispersion relations numerically as follows. We first search for a pair $\left(\beta_{0}, \omega_{0}\right)$ at which $P_{i}^{\epsilon_{0}}+P_{e}^{\epsilon_{1}} \Gamma$ has an eigenvalue that is practically zero: $\lambda\left(\beta_{0}, \omega_{0}\right) \approx 0$. Then we increment $\beta$ to $\beta_{1}$ and search in a complex vicinity of $\omega_{0}$ for a value $\omega_{1}$ such that $\lambda\left(\beta_{1}, \omega_{1}\right) \approx 0$. We continue to increment $\beta$, and in this way trace out a curve $\omega(\beta)$ represented by the computed points $\left(\beta_{n}, \omega_{n}\right)$ such that $\lambda\left(\beta_{n}, \omega_{n}\right) \approx 0$. To find the value $\omega_{n}$, we simply compute the minimum of the smallest eigenvalue $\lambda(\beta, \omega)$ of $P_{i}^{\epsilon_{0}}+P_{e}^{\epsilon_{1}} \Gamma$ as $\omega$ varies over a grid about $\omega_{n-1}$, keeping $\beta$ fixed at $\beta_{n}$, and then refine the search if necessary. We intend to develop a more efficient gradient search method for future investigations. In the examples in subsections 7.1 and 7.2 , we find the initial pair $\left(\beta_{0}, \omega_{0}\right)$ by taking $\beta_{0}=0$ and computing the minimum eigenvalue of $P_{i}^{\epsilon_{0}}+P_{e}^{\epsilon_{1}} \Gamma$, using MATLAB, on a grid of real values of $\omega$ in the interval $(0,1)$. An initial bound state was easy to find. In the example of subsection 7.3, we knew to search in the vicinity of a spike in the transmission graph that we had computed in [2].

To show that $\lambda(\beta, \omega)$ actually achieves a value of zero, it suffices to fix $\beta$ and compute (numerically) a positive winding number of $\lambda$ as an analytic function of $\omega$ about some small closed curve in the complex $\omega$-plane. We perform this verification at selected points on the dispersion relations. We also check numerically that the alternative (part (2)) in Theorem 5.1 does not hold.

To compute the eigenvalues of the boundary-integral operator $P_{i}^{\epsilon_{0}}+P_{e}^{\epsilon_{1}} \Gamma$, we discretize the integral system using quadratic basis elements for the fields and pointsampling of the equations. Complete details of these calculations are presented in [2]. Once we have obtained a numerical solution to $\left(P_{i}^{\epsilon_{0}}+P_{e}^{\epsilon_{1}} \Gamma\right) \boldsymbol{\psi}=\boldsymbol{\phi}$, we compute the scattering and bound $(\phi=0)$ states using Green's identities (3.4) and (3.5).

In all three examples, we study electrically polarized fields with $\epsilon_{0}=1$ and $\epsilon_{1}=12$.

In the figures, the fields in the crystals are represented by contour plots of their magnitudes. White represents the maximal amplitude, and black represents an amplitude of zero. One $y$-period is shown, with the $x$-direction truncated outside the support of the crystal slab.

7.1. A single string of rods. (Figure 7.1.) Our first example is a good illustration of the connection between dispersion relations, bound states, and resonant scattering phenomena. The period of our dielectric structure consists of a single rod in air, so that the crystal slab degenerates into a string of rods running in the $y$-direction. 
1. $\beta=0$; bound state frequency $\omega=W(0) \approx 0.6691$.

Bound state

Nearby scattering state

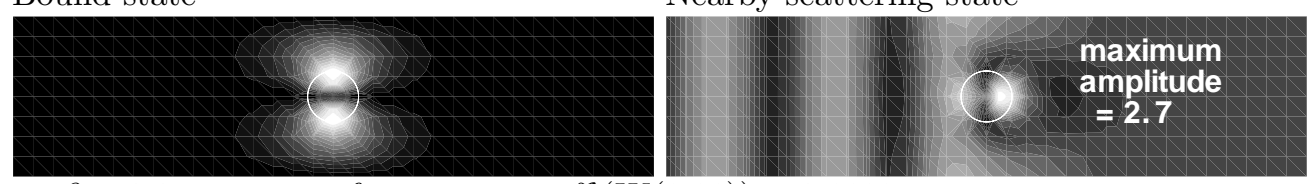

2. $\beta=0.01$; resonant frequency $\omega=\Re(W(0.01)) \approx 0.6690$.

Scattering state near $\omega=0.6690$

Scattering state away from $\omega=0.6690$
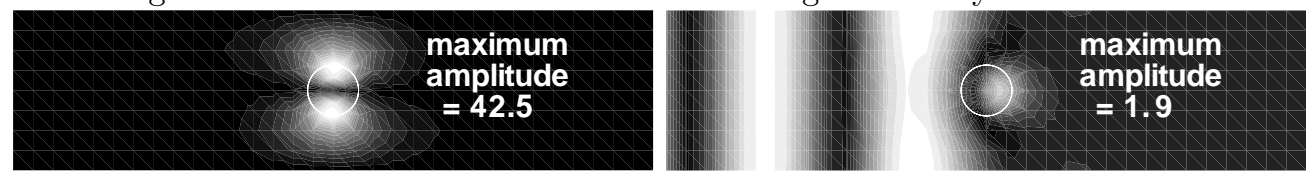

3. $\beta=0.12$; resonant frequency $\omega=\Re(W(0.12)) \approx 0.6601$.

Scattering state near $\omega=0.6601$

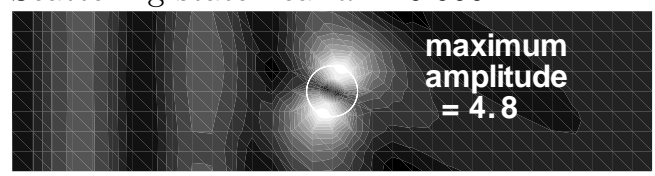

Scattering state away from $\omega=0.6601$

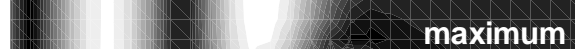

amplitude

$=2.0$

4.

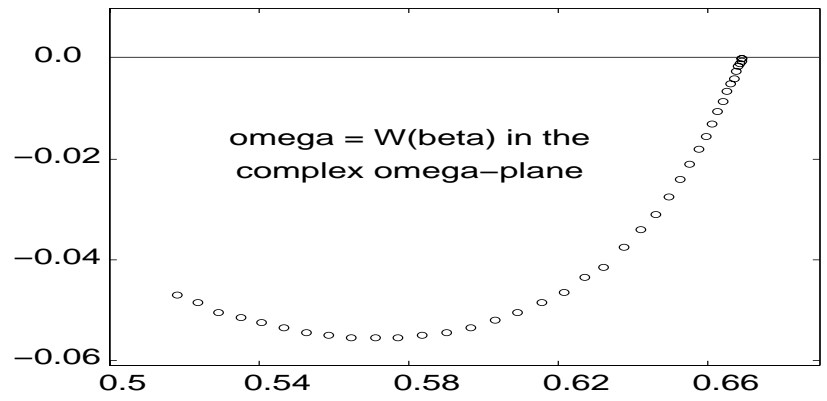

5.
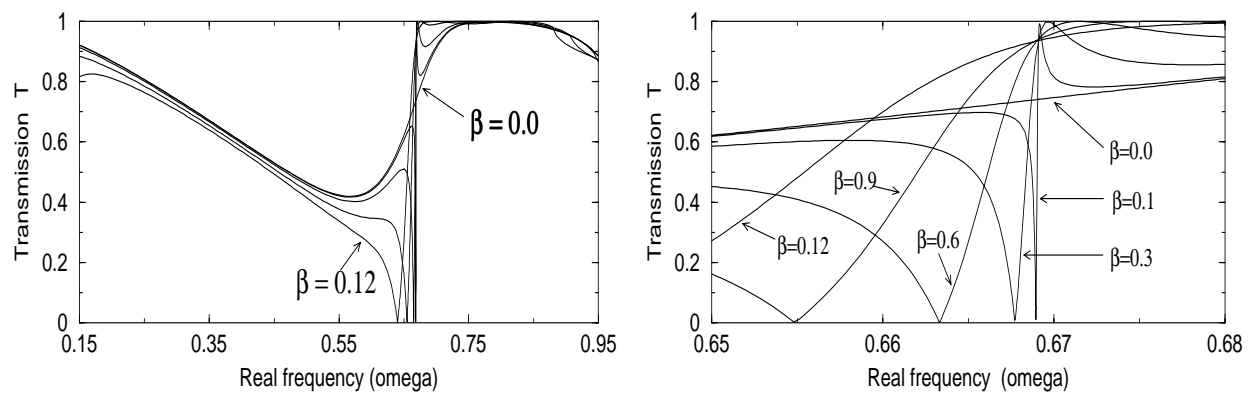

FIG. 7.1. In the contour plots, one period in $y$ of the crystal is shown, with the magnitude of the electrically polarized fields plotted. 1 . There is a bound state at $(\beta=0, \omega=W(0) \approx 0.6691)$. There is no unusually high amplification of an incident plane-wave source (of amplitude 1) for $\beta=0$ at frequencies $\omega$ near the bound-state frequency. 2,3. When $\Im(W(\beta))<0$, incident plane-wave sources are amplified in the rod at frequencies near the real part of $W(\beta)$. A smaller imaginary part corresponds to greater amplification. The field structure at resonant frequencies is similar to that of the bound state. 4. The dispersion relation $W(\beta)$ plotted in the complex $\omega$ plane for real values of beta from $\beta=0$ to $\beta=0.38$. 5. Spikes in the transmission coefficient near $\omega=\Re(W(\beta))$ for $\beta>0$, where $\Im(W(\beta))<0$. 
We find numerically a $y$-periodic (this means $\beta=0$ ) bound state at $\omega \approx 0.6691$. This is a standing wave that exists in the absence of any sources. However, as $\beta$ moves away from zero, the bound state disappears and the dispersion relation $\omega=W(\beta)$ enters the lower-half complex $\omega$-plane (Figure 7.1(4)). In place of a bound state, we find instead resonant scattering states at real frequencies near the real part $\Re(W(\beta))$ of the complex resonant frequency on the dispersion relation. These states are sustained by plane-wave source fields that are amplified as they experience resonant scattering within the dielectric structure. Coinciding with these large fields are anomalies (spikes) in the transmission coefficient ${ }^{2} T(\beta, \omega)$ for real values of $\omega$ near $\Re(W(\beta)$ ), for a fixed value of $\beta$ (see Figure 7.1(5)). These spikes consist of a drop to $0 \%$ transmission $(T=0)$ to the left of the frequency of the periodic bound state that exists at $\beta=0$, followed by a sharp increase to $100 \%$ transmission $(T=1)$ to the right. As $|\beta|$ decreases to zero, the width of the spike decreases and the resonant amplification of the scattering fields increases. The phenomena become more and more localized about the bound-state frequency. At $\beta=0$, the transmission anomaly and resonant behavior disappear, and we have in their place the bound state.

We find that, for $\omega_{0} \approx 0.6691, T\left(\beta, \omega_{0}\right) \approx 0.935$ for values of $\beta$ near but not equal to zero (see Figure 7.1(5)). However, continuing our calculation of the curve $T(0, \omega)$ through $\omega=\omega_{0}$ gives $T\left(0, \omega_{0}\right) \approx 0.739$. Thus we demonstrate numerically that $\lim _{\beta \rightarrow 0} T\left(\beta, \omega_{0}\right) \neq \lim _{\omega \rightarrow \omega_{0}} T(0, \omega)$, so that we cannot define the transmission coefficient continuously at $\left(0, \omega_{0}\right)$. This observation strengthens our belief that there is a bound state at $\left(0, \omega_{0}\right)$, that is, that $\Im(W(0))$ is indeed exactly zero.

From Figure 7.1(4), the dispersion relation evidently has the form

$$
\omega=\omega_{0}+a(\beta) \beta^{2},
$$

where $a$ is analytic and $a(0) \neq 0 .(W(\beta)$ is symmetric about $\beta=0$, as the Green's functions at $\pm \beta$ have the same set of modes, and the structure is symmetric in $y$.)

7.2. Surface waves. (Figure 7.2.) We investigate waves on the surface of a semi-infinite photonic crystal. We seek waves at the interface between the left halfplane containing air and the right half-plane filled with a square lattice of circular rods. If we consider frequencies that cannot propagate through the infinite crystal, or bandgap frequencies, it is reasonable to approximate the semi-infinite crystal by a finite slab several rods thick (truncated in the $x$-direction but still periodic in $y$ ). To capture surface waves at bandgap frequencies, we place a defect on the left surface of the slab by making the first rod much larger than the others.

There appears to be a periodic $(\beta=0)$ bound state at $\omega \approx 0.401$, as the smallest eigenvalue of $P_{i}^{\epsilon_{0}}+P_{e}^{\epsilon_{1}} \Gamma$ is practically zero there. The field is localized at the defective surface of the slab (see Figure 7.2(1)) - it is a standing surface wave.

As $\beta$ moves away from zero, the surface wave disappears, and we obtain a dispersion relation $\omega=W(\beta)$ with a very small negative imaginary part. At about $\beta \approx 0.345$, however, the relation enters a regime in which $|W(\beta)|<\min _{m \in \mathbb{Z}}(|\beta+m|)$, which is the scenario in which all modes of the Green's function decay in $|x|$ and the exterior medium admits no traveling waves. We deduce from Theorem 5.1 (or Theorem 5.2) that this part of the dispersion relation is necessarily real and is therefore a

\footnotetext{
${ }^{2}$ Our transmission coefficient is the square root of the ratio of the energy transmitted through the slab on the right to the energy of a plane-wave source field $\exp \left(i \sqrt{\epsilon_{0} \omega^{2}-(m+\beta)^{2}} x+i(m+\beta) y\right)$ incident upon the slab from the left. Details of how we calculate the transmission coefficient are given in [2].
} 
1. A bound state at $\beta=0, \omega \approx 0.401$ : a standing surface wave.

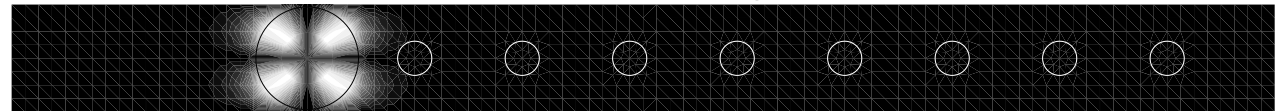

2. A scattering state for $\beta=0$ at a frequency near that of the bound state in (1). mexi

ampli is $=2$.

3. A scattering state at $\beta=0.23, \omega \approx 0.368$, where $|\Im(W(\beta))|$ is very small.

maximm

amplitude

$=25.8$

4. A scattering state at $\beta=0.28, \omega \approx 0.358$, near $\max |\Im(W(\beta))|$.

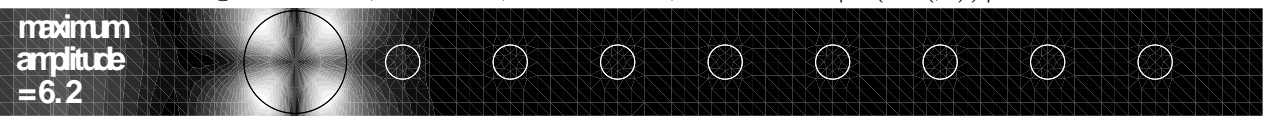

5. A bound state at $\beta=0.40, \omega \approx 0.335$ : a traveling surface wave.
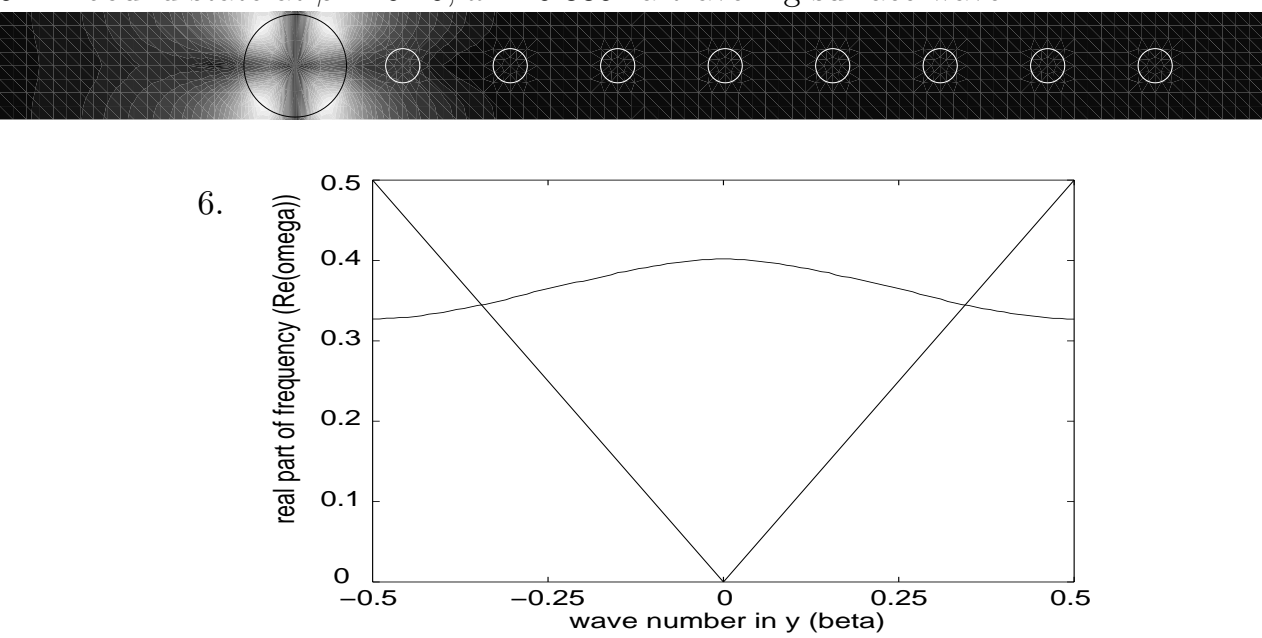

7.

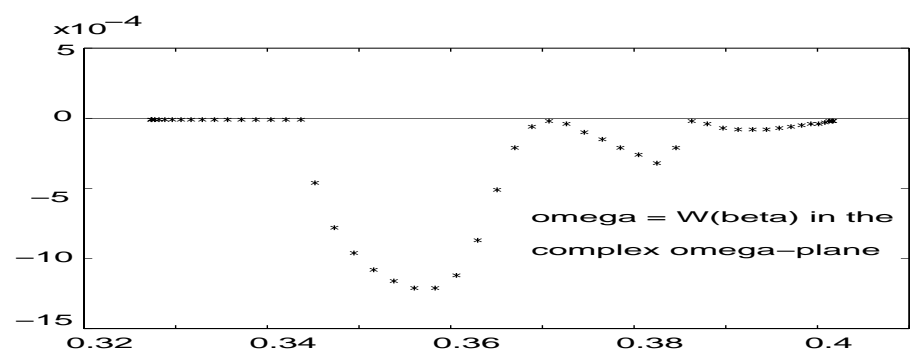

FIG. 7.2. A crystal slab with a defect on the left surface, where one rod is bigger than the rest. In the contour plots, one period in $y$ of the crystal is shown, with the magnitude of the electrically polarized fields plotted. 1,2. There is a periodic $(\beta=0)$ bound state, which is a standing surface wave localized in the large rod at the surface of the crystal slab. At frequencies near that of the surface wave, incident plane-wave sources are not amplified in the rod. 3,4. When $\Im(W(\beta))<0$, incident plane-wave sources (with amplitude 1 ) at frequencies near $\Re(W(\beta)$ ) resonate in the first rod. 5. A bound state at parameter values on the dispersion relation, where $\omega=W(\beta)$ is necessarily real and the strip admits no scattering (extended) states. $6 . \Re(W(\beta))$ plotted against $\beta$. $\Im(W(\beta))=0$ when $\Re(W(\beta))<|\beta|(0.345<|\beta|<0.5)$. 7. $\omega=W(\beta)$ plotted in the complex $\omega$-plane. 
dispersion relation for $x$-localized fields. In fact, in this regime, there are no scattering (extended) states in the strip (see Remark 2 following Theorem 5.1).

We therefore demonstrate the existence of a dispersion relation for waves traveling along the surface of a photonic crystal slab. Our choice of eight rods to approximate the semi-infinite crystal appears to be sufficient: Increasing the number of rods results in no appreciable difference in the calculated curve. Thus, we are led to believe that the dispersion relation for the semi-infinite crystal exists and that the dispersion relation for the slab is a good approximation.

As in the case of the single string of rods in the previous example, we see again how the complex part of the dispersion relation, which is very close to the real axis, affects the scattering states at nearby real frequencies. We do not find anomalies in the transmission as we did in that example; however, we believe that they are there but are too sharp to be detected numerically because of the small size of the imaginary part of the dispersion relation and the thickness of the slab.

7.3. A channel defect. (Figure 7.3.) In [2], we studied the effect that a periodic channel through a photonic crystal slab has on the transmission coefficient and the structure of the scattering states. Our intention was to study resonant behavior at bandgap frequencies and modes propagating through the channel; however, we also found intriguing sharp transmission spikes and resonant scattering fields in near-fulltransmission frequency regions. The phenomena are similar in behavior to those in our previous two examples, and a similar analysis provides us with a better mathematical understanding of them.

The $(\beta, \omega)$ pairs at which resonant behavior and transmission anomalies occur for the crystal with a periodic channel are described by a dispersion relation $\omega=$ $W(\beta)$, which we calculate numerically. ${ }^{3}$ We demonstrate in [2] that both phenomena disappear as the width of the channel decreases and the slab returns to its perfect structure. In the present study, we find that, in place of resonant scattering states, the perfect slab admits bound states, also described by a dispersion relation.

Our numerical calculations give us dispersion relations that lie practically on the real $\omega$-axis. However, they may have a small negative imaginary part that we cannot resolve numerically. Based on our findings in the previous two examples, we conjecture that the relation is identically real for the perfect slab (there is no resonant amplification, and there are no transmission spikes) and that it has a very small negative imaginary part for the slab with a periodic channel (there are highamplitude resonances and very sharp transmission spikes).

The structure of the resonant fields near $\omega=\Re(W(\beta))$ when $\Im(W(\beta))$ is small resembles the field of a nearby bound state, if such a bound state exists. See Figure 7.3(1) and (3), which shows the $y$-directional structure of a bound state and a resonant scattering state. A typical scattering state at small values of $\beta$ exhibits the structure of an $x$-directional interference pattern, a point we discuss in [2].

In summary, we have a plausible explanation for the channel-induced resonant behavior that we observed in [2]: There is a dispersion relation $\omega=W(\beta)$ for bound states traveling along the perfect crystal slab. When a periodic channel is introduced, the new (perturbed) relation $\omega=W(\beta)$ gives resonant frequencies with a small imaginary part. These are responsible for the observed buildup of large fields in the slab and for the transmission anomaly near $\omega=\Re(W(\beta))$. In the limit of zero imaginary

\footnotetext{
${ }^{3}$ In [2] , we scaled the frequency in this example by $1 / 6$ to compare with the scale of the finer period of one rod. We did not make that scaling here.
} 
1. a.

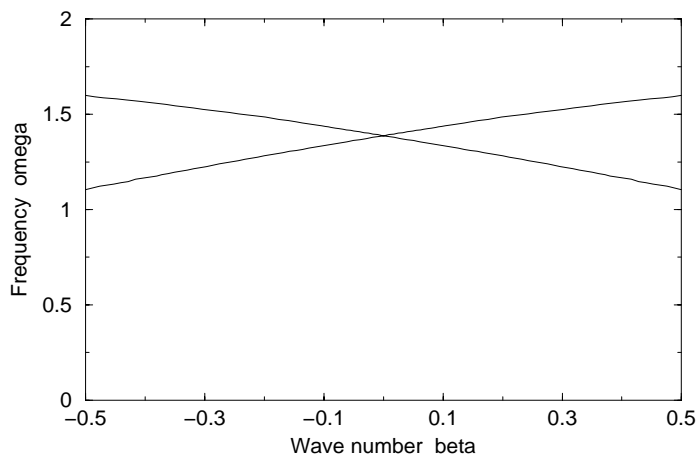

2. a.

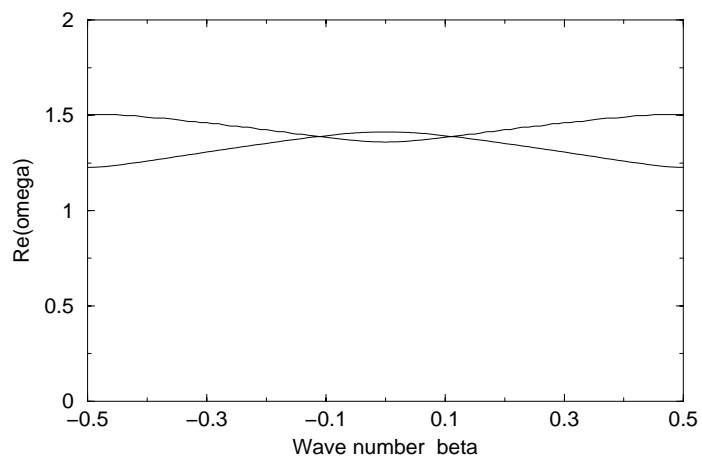

3. a.

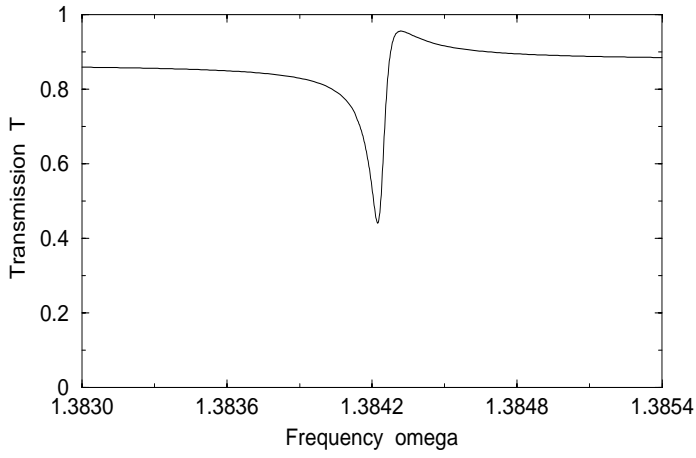

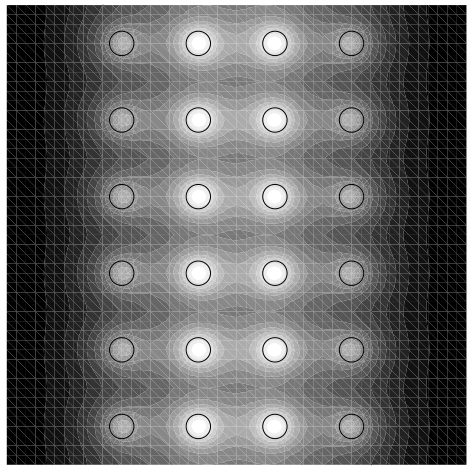

b.

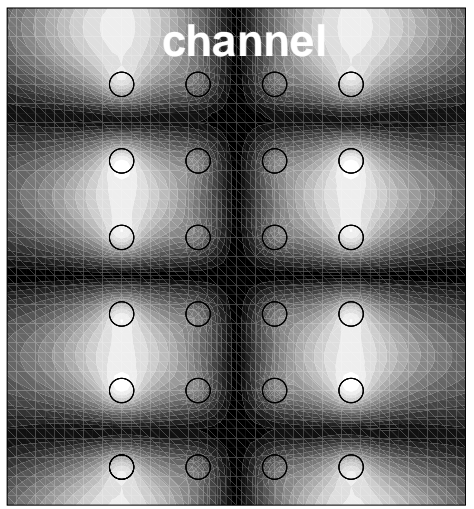

b.

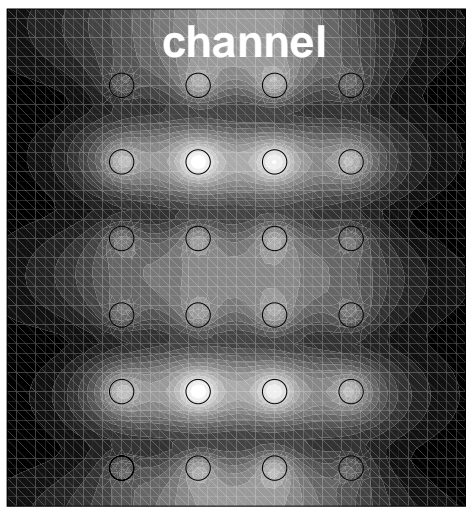

FIG. 7.3. In the contour plots, a period in $y$ of the crystal is shown, and the strip is truncated in the x-direction. The magnitude of the electrically polarized fields is plotted. 1.a. Branches of the dispersion relation for a perfect square-lattice crystal four rods thick. 1.b. The bound state at $\beta=0.1$ and $\omega \approx 1.434$. 2.a. Branches of the real part of the dispersion relation for the crystal with a periodic channel containing an extra half-period of space after every six rows of rods. 2.b. A resonant scattering state at $\beta=0.5$ and $\omega \approx 1.224$. 3.a. An anomaly in the transmission coefficient for $\beta=0.1$. 3.b. A resonant scattering state at $\beta=0.1$ and $\omega \approx 1.3842$ (slightly to the left of where the two branches of the dispersion relation cross, on the lower branch). The amplitude of the field in the crystal reaches about 49 times the amplitude of the incident field. 
part, the anomaly occurs at a single point (the point of the bound state) and is not observed through the numerical scattering experiment.

The numerical evidence indicates that the slab with a periodic channel supports resonant frequencies that converge to bound state frequencies as the channel closes up. A similar phenomenon is known for Helmholtz resonators, in which resonant frequencies of a cavity with an opening converge to the bound state frequencies of the closed cavity as the opening disappears (Beale [6]).

8. Appendix. Let $D$ be a domain whose boundary $C=\partial D$ is a closed curve of class $C^{2}$ with arclength parameterization $\gamma(t), t \in[a, b]$, and outward-pointing normal vector $n(t)$ at $\gamma(t)$. For sufficiently small values of $\rho>0$, the function $\gamma_{\rho}(t)=$ $\gamma(t)-\rho n(t)$ parameterizes a $C^{1}$ closed curve $C_{\rho}$ contained in the interior of $D$.

The Green's kernel $G(\mathbf{r})$ (or its derivatives) takes a function $\phi$ on $C$ to a function $\psi_{\rho}$ on $C_{\rho}$ by

$$
\psi_{\rho}(\mathbf{r})=\int_{C} G(\hat{\mathbf{r}}-\mathbf{r}) \phi(\mathbf{r}) d s(\mathbf{r})
$$

where $\hat{\mathbf{r}} \in C_{\rho}$. This map can be realized as a map taking a function on $[a, b]$ to another by recycling notation and writing

$$
\psi_{\rho}(t)=\int_{a}^{b} G\left(\gamma_{\rho}(t)-\gamma(s)\right) \phi(s) d s
$$

We make the identifications $H^{1}(C) \equiv H^{1}([a, b])$ and $L^{2}(C) \equiv L^{2}([a, b])$.

1. The singular part of the integral kernel $G(\gamma(t)-\gamma(s))$ is $\log |t-s|$, and the singular part of its derivative, $d / d t \log |t-s|$, which has the Hilbert-transform singularity, is a principal-value integral. Both are bounded operators from $L^{2}$ to $L^{2}$, so $G(\gamma(t)-\gamma(s))$ is a bounded operator from $L^{2}$ to $H^{1}$. $G\left(\gamma_{\rho}(t)-\right.$ $\gamma(s))$ and $d / d t G\left(\gamma_{\rho}(t)-\gamma(s)\right)$ are regularizations of these singular kernels, so $G\left(\gamma_{\rho}(t)-\gamma(s)\right)$ converges to $G(\gamma(t)-\gamma(s))$ as $\rho \rightarrow 0$ as bounded operators from $L^{2}$ to $H^{1}$.

2. By the theory of the double-layer potential and the Plemelj formula, the integral kernels

$$
\frac{\partial G\left(\gamma_{\rho}(t)-\gamma(s)\right)}{\partial n(\gamma(s))}, \quad-\frac{\partial G\left(\gamma_{\rho}(t)-\gamma(s)\right)}{\partial n(\gamma(t))},
$$

as applied to $L^{2}$, are a regularization of their limiting form as $\rho \rightarrow 0$, which is $1 / 2$ the identity operator plus a weakly singular integral kernel.

3. Using the identity

$$
\frac{\partial^{2} G(\hat{\mathbf{r}}-\mathbf{r})}{\partial n(\hat{\mathbf{r}}) \partial n(\mathbf{r})}+\frac{\partial^{2} G(\hat{\mathbf{r}}-\mathbf{r})}{\partial t(\hat{\mathbf{r}}) \partial t(\mathbf{r})}=-n(\hat{\mathbf{r}}) \cdot n(\mathbf{r})\left(\partial_{x}^{2}+\partial_{y}^{2}\right) G(\hat{\mathbf{r}}-\mathbf{r})
$$

and the Helmholtz equation

$$
\left(\partial_{x}^{2}+\partial_{y}^{2}\right) G(\hat{\mathbf{r}}-\mathbf{r})+\epsilon \omega^{2} G(\hat{\mathbf{r}}-\mathbf{r})=0 \quad \text { for } \quad \hat{\mathbf{r}} \neq \mathbf{r},
$$

we can rewrite the kernel $\partial^{2} G(\hat{\mathbf{r}}-\mathbf{r}) / \partial n(\hat{\mathbf{r}}) \partial n(\mathbf{r})$ for $\hat{\mathbf{r}} \notin \partial D$, applied to a 
function $\xi \in H^{1}$ :

$$
\begin{aligned}
& \int_{\partial D} \frac{\partial^{2} G(\hat{\mathbf{r}}-\mathbf{r})}{\partial n(\hat{\mathbf{r}}) \partial n(\mathbf{r})} \xi(\mathbf{r}) d s(\mathbf{r}) \\
& \quad=\int_{\partial D}\left(n(\hat{\mathbf{r}}) \cdot n(\mathbf{r}) \epsilon \omega^{2} G(\hat{\mathbf{r}}-\mathbf{r}) \xi(\mathbf{r})+\frac{\partial G(\hat{\mathbf{r}}-\mathbf{r})}{\partial t(\hat{\mathbf{r}})} \frac{d \xi}{d t(\mathbf{r})}(\mathbf{r})\right) d s(\mathbf{r}) .
\end{aligned}
$$

The kernel $\partial G / \partial t$ converges to a principal-value kernel as $\hat{\mathbf{r}} \rightarrow \partial D$, and, since $d \xi / d t(\mathbf{r}) \in L^{2}$, we see that the operator with kernel $\partial^{2} G(\hat{\mathbf{r}}-\mathbf{r}) / \partial n(\hat{\mathbf{r}}) \partial n(\mathbf{r})$ converges to a bounded operator from $H^{1}$ to $L^{2}$ as $\hat{\mathbf{r}} \rightarrow \partial D$.

\section{REFERENCES}

[1] S. Venakides, M. A. Haider, and V. Papanicolaou, Boundary integral calculations of twodimensional electromagnetic scattering by photonic crystal Fabry-Perot structures, SIAM J. Appl. Math., 60 (2000), pp. 1686-1706.

[2] M. A. Haider, S. P. Shipman, and S. Venakides, Boundary-integral calculations of twodimensional electromagnetic scattering in infinite photonic crystal slabs: Channel defects and resonances, SIAM J. Appl. Math., 62 (2002), pp. 2129-2148.

[3] S. Fan, P. R. Villeneuve, and J. D. Joannopoulos, Channel-drop filters in photonic crystals, Optics Express, 3 (1998), pp. 4-11.

[4] A. Krishnan, T. Thio, T. J. Kim, H. J. Lezec, T. W. Ebbessen, P. A. Wolff, J. Pendry, L. Martin-Moreno, and F. J. Garcia-Vidal, Evanescently coupled resonance in surface plasmon enhanced transmission, Optics Commun., 200 (2001), pp. 1-7.

[5] S. G. Tikhodeev, A. L. Yablonskit, E. A. Muljarov, N. A. Gippius, and T. Ishihara, Quasiguided modes and optical properties of photonic crystal slabs, Phys. Rev. B, 66 (2002), 045102 .

[6] J. T. Beale, Scattering frequencies of resonators, Comm. Pure Appl. Math., 26 (1973), pp. 549-563.

[7] A. P. Calderón, Boundary-value problems for elliptic equations, in Outlines Joint Sympos. Partial Differential Equations (Novosibirsk, Russia), Acad. Sci. USSR Siberian Branch, Moscow, 1963, pp. 303-304.

[8] R. T. SEeley, Singular integrals and boundary value problems, Amer. J. Math., 88 (1966), pp. 781-809.

[9] V. S. Ryaben'KiI, Boundary equations with projections, Russian Math. Surveys, 40 (1985), pp. $147-183$.

[10] J.-C. NÉDÉLEC, Acoustic and Electromagnetic Equations, Springer-Verlag, New York, 2001.

[11] C. Müller, Foundations of the Mathematical Theory of Electromagnetic Waves, SpringerVerlag, New York, 1969.

[12] D. L. Colton And R. Kress, Inverse Acoustic and Electromagnetic Scattering Theory, 2nd ed., Springer-Verlag, New York, 1998.

[13] D. L. Colton And R. Kress, Integral Equation Methods in Scattering Theory, WileyInterscience, New York, 1983. 